\title{
A multifaceted hospital-wide intervention increases hand hygiene compliance
}

\author{
B Patel, H Engelbrecht, H McDonald, V Morris, W Smythe \\ Dr Bhavna Patel is the Chief Executive Officer at Groote Schuur Hospital, Cape Town, South Africa. Srs Heather Engelbrecht, Vida Morris \\ and Heidi McDonald are all registered nurses, responsible for the management of infection prevention and control at Groote Schuur Hospital. \\ Mr Wynand Smythe is registered as a PhD candidate in the Department of Clinical Pharmacology, Faculty of Health Sciences, University of Cape \\ Town, and works as a research pharmacist in the Clinical Research Centre at UCT.
}

Corresponding author: B Patel (bhavna.patel@westerncape.gov.za)

\begin{abstract}
Background. Hand hygiene is an important and basic practice that should be used by all healthcare staff to protect both themselves and their patients against infection. Unfortunately hand hygiene compliance remains poor.

Objective. To show an improvement in hand hygiene compliance using a multifaceted approach.

Methods. This was a quasiexperimental pre-post intervention study design with a number of standardised interventions to promote hand hygiene. The World Health Organization hand hygiene multimodal (five-step) intervention approach was used. The study ran from June 2015 to August 2015 in 11 selected wards of a 975-bed tertiary and quaternary care public hospital (Groote Schuur Hospital, Cape Town, South Africa). The outcome was to assess improvement in hand hygiene compliance monthly over the 3 months, compared with nonintervention wards and compared with the wards' own performance measured in 2014. The study included both descriptive and analytical components.

Results. Post intervention, hand hygiene compliance showed a statistically significant improvement for before patient contact from $34 \%$ in 2014 to $76 \%$ in $2015(p<0.05)$ and for after patient contact from $47 \%$ in 2014 to $82 \%$ in $2015(p<0.05)$.

Conclusion. The intervention improved hand hygiene compliance and can easily be replicated in other wards, resulting in sustaining a culture of hand hygiene improvement and behavioural change throughout the hospital.
\end{abstract}

S Afr Med J 2016;106(4):335-341. DOI:10.7196/SAMJ.2016.v106i4.10671

Hospital-acquired infections remain a global concern, with prevalence rates in the USA ranging from $3.5 \%$ to $9.9 \%,{ }^{[1]}$ almost one-third of infections being preventable. In South Africa (SA), about one in seven patients entering health facilities may be at risk of developing a hospitalacquired infection. This results in significant morbidity and mortality, pressures on the need for intensive care beds, and the direct and indirect costs of these infections. ${ }^{[2]}$ Factors contributing to this risk include indiscriminate use of antibiotics over the years, resulting in drugresistant organisms and more recently multidrug-resistant organisms. Patients may be discharged by the time the infection presents and it can then also spread in the community, making it difficult to distinguish between community-acquired and hospital-acquired infections. As there is no monitoring of these infections the prevalence cannot be measured, but it is estimated that approximately $10-25 \%$ of patients admitted to SA hospitals may acquire an infection. ${ }^{[3]}$

Hand washing with soap and water is considered to be hygienic. However, the use of antiseptic agents for cleansing hands emerged when a French pharmacist demonstrated their benefits when managing corpses, and in 1825 suggested their use to doctors attending to patients with contagious diseases. Semmelweis first showed that the cleansing of hands with an antiseptic agent may reduce healthcare-associated transmission of contagious diseases. ${ }^{[4]}$

Organisms that cause hospital-acquired infections are usually transmitted through contact with the patient by doctors, nurses, physiotherapists and other hospital personnel. This can be through skin-to-skin contact, direct spread for droplets $>5$ microns in size and airborne spread for droplets $<5$ microns in size. ${ }^{[2]}$ Besides general safety precautions, hand hygiene has been recommended as the single most important measure in preventing hospital-acquired infections. The Centers for Disease Control (CDC) guidelines suggest hand washing with antimicrobial soap between every patient contact and before and after performing invasive procedures. ${ }^{[4]}$

Despite these guidelines, hand hygiene compliance is frequently poor. Healthcare workers in intensive care units (ICUs) have been reported as failing to wash their hands more than half of the recommended times, and even when performed, the procedure was inadequate. ${ }^{[5]}$ Less than $50 \%$ compliance has been found when comparing different hospital wards among different professional categories of healthcare workers. ${ }^{[6]}$ Compliance also decreased depending on the patient demand and intensity of care. Reasons for failure to comply noted by healthcare workers included skin irritation, inaccessible supplies, interference with work, forgetfulness, emergency care, understaffing and high workload. ${ }^{[6]}$ A 2009 multicentre study found that baseline compliance in an ICU was $26 \%$, with $36 \%$ for non-ICU settings. ${ }^{[7]}$ A systematic review of 96 studies published before 2009 reflected an average compliance of $40 \%{ }^{\left[{ }^{[8]}\right.}$

The agents used for washing or disinfecting hands should be able to eliminate transient flora, but micro-organisms can only be killed using antiseptic agents. Alcohol-based preparations have the more rapid action of all antiseptics, and are more convenient than others because of their rapid evaporation and spreading quality. ${ }^{[6]}$

Increasing compliance in the use of these agents for hand washing remains a problem. Educational programmes, communication campaigns, visual reminders, audits and constant supervision and monitoring must be employed to improve compliance. Such interventions have changed cultural behaviours, and institutions have been successful in sustaining these improvements. ${ }^{[6,9,10]}$

Materials used to implement such improvements include the $\mathrm{CDC}^{[4]}$ and World Health Organization (WHO) guidelines. ${ }^{[1]}$ 
Infrastructural requirements such as the availability and accessibility of hand sanitisers, alcohol-based rub solutions and dispensers must also be in place. An increase in hand hygiene compliance from $23 \%$ to $48 \%$ was demonstrated following the introduction of an easily accessible alcohol-based waterless sanitiser dispenser. ${ }^{[12]}$ Others have also looked at factors that affect ease of access and result in improved hand hygiene performance, e.g. the placement, proximity, cost, staff acceptance, durability, maintenance and environmental

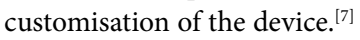

Groote Schuur Hospital (GSH), Cape Town, SA, is a 975-bed specialist and subspecialist hospital. Antimicrobial resistance has increased over the past 2 - 3 years, particularly resistance to the carbapenems. Globally, the dissemination of New Delhi metallo-beta-lactamase1-containing Gram-negative bacteria and carbepenem-resistant Klebsiella pneumoniae increases fears of an international meltdown in treating infections. For some organisms the last-line antimicrobial agents are also starting to show some resistance, which could spark an era of morbidity and mortality that modern medicine and technology will not be able to control. One of the best preventive measures would be to decrease the spread of infection through practising basic precautionary measures such as hand washing. A baseline audit in 2014 on hand hygiene at GSH reflected an average performance of $34 \%$ for the hospital.

This study was a multifaceted, hospital-wide hand hygiene intervention using the WHO approach and developing a model to sustain a change in culture in our institution.

\section{Objective}

To measure whether there was an improvement in hand hygiene compliance before and after contact with the patient over the period June - August 2015 in 11 selected wards at GSH, while implementing hospital-specific standardised interventions including the appointment of a ward hand hygiene champion, an educational programme, posters, presentations and regular audits and feedback. Compliance was measured monthly over the 3 months and compared with non-intervention control wards and the wards' own performance during the previous year.

\section{Methods}

\section{Approval}

The study was approved by the Human Research Ethics Committee, Faculty of Health Sciences, University of Cape Town, South Africa (HREC/REF:077/2015). Institutional ethics approval was granted by the hospital research ethics committee. Each participant was asked to give written informed consent at the start of the educational programme.

\section{Study design}

This was a quasiexperimental pre-post interventional design conducted over the 3 months June - August 2015 to statistically compare the practice of hand hygiene in 11 selected educational intervention wards over the study period with similar control wards that did not receive the intervention and with the wards' own performance during the previous year. Descriptive and analytical components were included.

\section{Study population and sampling}

All staff working in the selected wards across all disciplines, irrespective of their professional category or rank, were included. A sample size of 146 for the intervention group was calculated using a $95 \%$ confidence interval, $80 \%$ power and an estimated compliance improvement rate of $50 \%$.

\section{Information, consent and procedures}

Each ward selected a hand hygiene champion who facilitated the activities of the intervention. Using the WHO hand hygiene multimodal (five-step) intervention approach, the study procedure included the following:

1. A pre-study needs-assessment questionnaire ensured that all infrastructural and consumable requirement needs were met.

2. A hospital-specific standardised training presentation was prepared and the champions were trained on how to conduct the presentation at least once per week to ensure that all ward staff received the training. Participants were given an information sheet and consent form at the start of the educational session.

3. Posters were placed above the basins in the ward and the champions were asked to provide ad hoc in service training (Appendix 1) based on the WHO 'five moments of hand hygiene' intervention approach.

4. Standardised hand hygiene audit tools and observation methods were used to assess the performance of the staff for adherence to the five moments of hand hygiene, while working with the patients. This was done once per month over the 3-month period by the independent infection control team. These tools provided the data from which compliance was measured. The same tool was used in the previous year, from which the baseline measure of compliance was determined.

5. Monthly feedback was provided to the hand hygiene champions, and performance graphs were circulated for use in the educational sessions.

Furthermore, there was leadership and commitment from hospital management to ensure that these measures would assist in creating a continuous culture of improvement and behaviour change among the staff.

\section{Statistical analysis}

The primary outcome was hand hygiene compliance, which was calculated by dividing the number of hand hygiene episodes by the number of potential opportunities. This measure was calculated before and after contact with patients over both years. Using the statistical package Statistica (version 12), factorial repeated measures analysis of variance (ANOVA) was applied to consider the effect of intervention and contact occasion, intervention ward and contact occasion, intervention and year on hand hygiene compliance.

\section{Resullts}

Over the period June 2015 - August 2015, the hand hygiene champions in the 11 wards trained 557 staff using the hospital-specific standardised educational presentation provided. Of these, 497 (89.2\%) gave written consent for participation. They comprised 110 medical doctors (22.1\%), 273 nursing staff $(54.9 \%)$ and 114 other staff (22.9\%), including cleaners, porters and allied health staff.

During the audits 497 observations were recorded over the 3 -month period. All the intervention wards showed an improvement in hand hygiene compliance from the 1st month to the 3rd month, from a combined average improvement from $55 \%$ to $73 \%$ for before patient contact and from $56 \%$ to $79 \%$ for after patient contact (Fig. 1). While all wards progressively improved over the 3 months, one of the two surgical wards, a gynaecological ward and theatre areas dipped in performance during the second month and also showed the smallest percentage improvement $(<10 \%)$ from the first to the third month. The greatest improvement (50\%) was seen in the ICU and the second surgical ward.

For these same intervention wards, the difference in hand hygiene compliance between the intervention and control wards was 


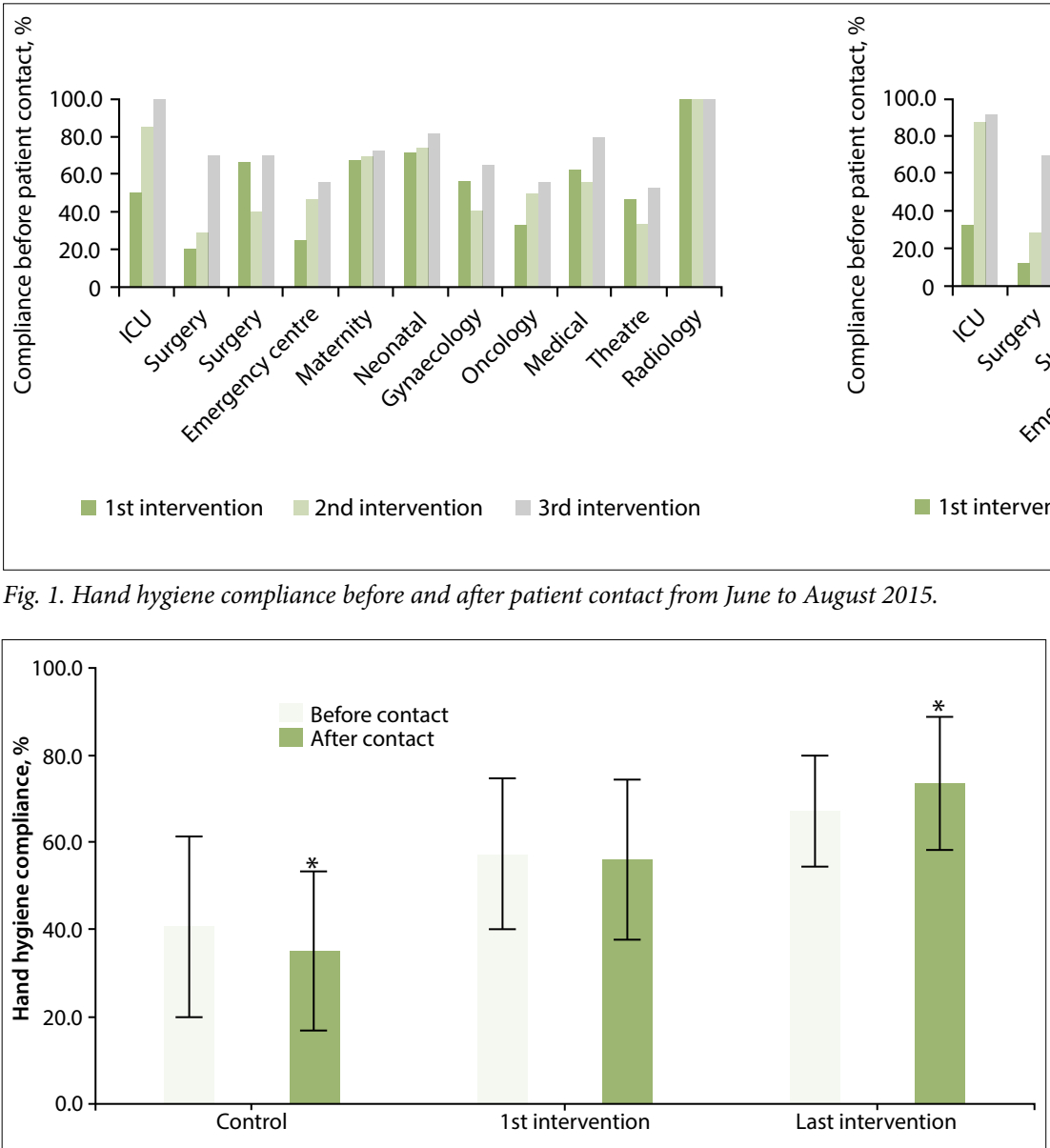

Fig. 2. Hand hygiene compliance between the intervention and control wards. Applying a factorial repeated measures ANOVA to consider the effect of intervention and contact occasion, intervention ward and contact occasion, intervention and year on hand hygiene compliance found a significant effect on hand hygiene compliance (ANOVA, $\mathrm{F}=2.74465, \mathrm{p}=0.037588$ ). Bars sharing a common symbol $\left({ }^{*}\right)$ are significantly different $(\mathrm{t}=3.04641, \mathrm{p}=0.005006)$.

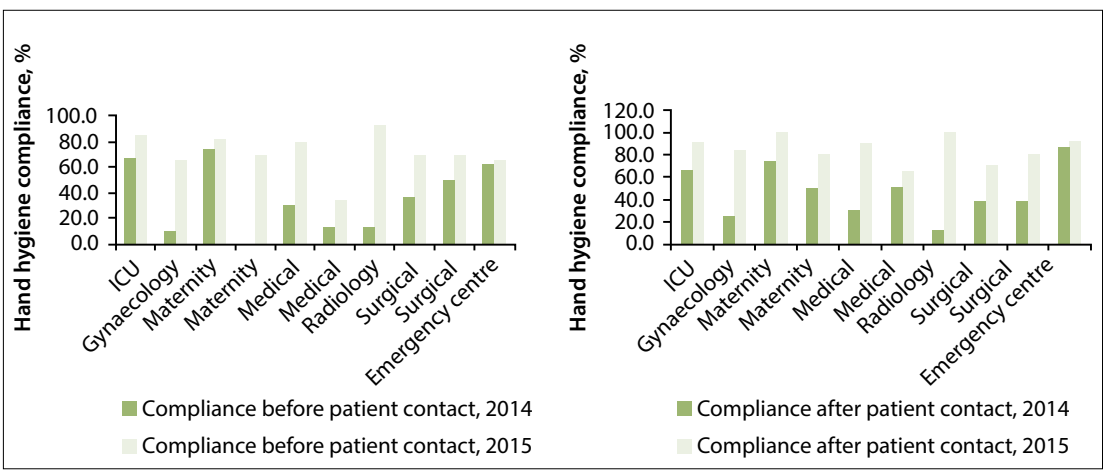

Fig. 3. Overall improvement in hand hygiene compliance in 2015 compared with 2014.

significant $(p<0.05)$ (Fig. 2). The greatest improvement was seen in the maternity, surgical and trauma wards.

The intervention wards also improved in compliance from their 2014 baseline performance to the 2015 post-intervention assessment by a combined average of $34 \%$ to $76 \%$ (range $3.5-80.5$ ) and $47 \%$ to $82 \%$ (range 4.5 - 87.5) for before and after patient contact, respectively (Fig. 3).
The nursing staff showed better compliance than the other healthcare categories and were more willing to be the ward champions. Doctors performed better when the hand hygiene champion was also a doctor.

Difficulties encountered by the hand hygiene champions included:

- Staff rotations and turnover as a result of doctor and nurse rotations and dependence on agency staff in some wards.

- Lack of support from the managers to release staff for training, mainly due to staff shortages to cover the clinical work, but to lack of commitment from managers in some instances.

- Keeping staff motivated. This problem was approached by changing the presentation to include stimulation in the form of additional learning.

- Time constraints for the champions to cover their normal daily duties and be the ward hand hygiene champion.

\section{Discussion}

Notable findings included the role of the hand hygiene champion in the ward, and the significant improvement in hand hygiene compliance over the 3-month period compared with similar control wards and the wards' own performance in 2014. While poor performance may be due to a lack of knowledge, the improvement can be attributed to the educational training and visual reminders, but mostly to the role that the champions played in motivating and constantly observing the hand hygiene practices. The results achieved by improving knowledge together with visual reminders, audits and regular feedback show that use of a multimodal approach should be promoted. Nurses were more compliant than doctors, but with a doctor as the hand hygiene champion, the training, supervision and 


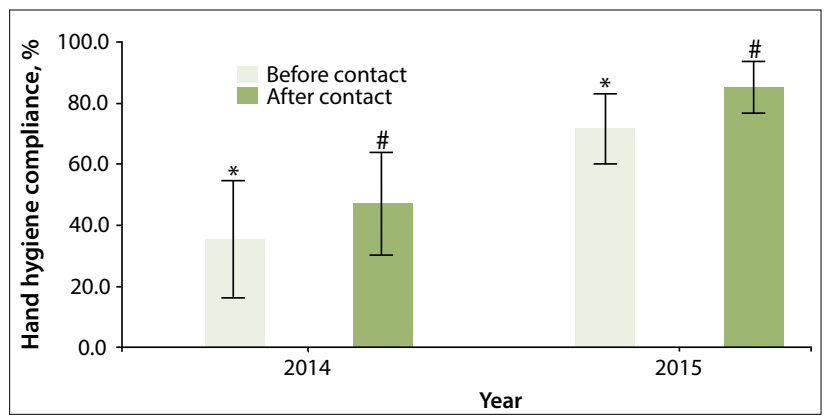

Fig. 4. Overall hand hygiene compliance in 2014 and 2015. Bars sharing a common symbol are significantly different $(\mathrm{t}=3.04641, \mathrm{p}=0.005006)$. Differences before patient contact $\left(^{*}\right) \mathrm{t}=-3.66521, \mathrm{p}=0.001771$; differences after contact (\#) $\mathrm{t}=-4.58698, \mathrm{p}=0.000229$.

compliance of other doctors were easier and better than when a nurse was appointed as the champion. This mind-set culture of assuming that the doctor is always right should be used to the advantage of such a programme and can be of value if combined with education on hospital-acquired infection rates and antibiotic stewardship, creating a comprehensive approach to patient management. A multidisciplinary team approach in the ward, together with such a comprehensive management system, could enhance the quality of patient care.

Standardising the educational material assisted the champions in presenting the same message in all areas and in every session, but they noted that staff who attended more than one session needed additional stimulation. Additional presentations should therefore be prepared for ongoing education and to retain staff motivation.

The challenge of sustaining the improved hand hygiene compliance and improving performance further was taken forward by the ward champions, who also served as role models for other wards where the same programme could be started. Unfortunately, owing to the lack of adequate data on specific ward infection rates, this improvement cannot be matched to clinical outcomes.

\section{Study limitations}

Despite the success of the programme, the study has limitations. The main limitation was the quasiexperimental design, where there may have been selection bias in the choice of the intervention wards, although an attempt was made to ensure that one ward was selected from every department at the hospital. Similarly, the control wards were selected to match the intervention wards in size, staff numbers and patient type. The sample size was also not the same for the pre- and post-intervention phases, because staff moved between wards. Randomisation of the wards and ensuring a stable sample was not feasible because the study was performed in one hospital in the hope of showing improved hand hygiene compliance using a programme that could be replicated in other wards. The intervention group received educational training from the selected champion for the area, which may have introduced bias. Although standardised educational material was provided for training, the champions could have interpreted the material differently and in-house supervision could also have been different in the different wards, depending on the respective champion.
Inter-observer bias was present because three different investigators performed the audits, but this small number and standardised audit tool reduced variability. However, it introduced a Hawthorne effect because staff were aware of being observed by the investigators' audits. Other confounding factors that could not be measured include the role of leadership and commitment from the hospital senior managers. This is evidenced by the number of hand hygiene promotional activities held at the hospital and the participation in a hand hygiene relay event on International Hand Hygiene Day. Such awareness could also introduce bias in compliance with hand hygiene and hence the outcome of the study. Control for this was beyond the scope of this study, and we had to rely on the experience of the staff concerned. Other studies ${ }^{[5,6,8-10]}$ have also shown an improvement in hand hygiene compliance, with some using the WHO multimodal approach, but this study is different in that it achieved similar results over a short period of time, using ward staff working under severe resource constraints. While it can be argued that the results could be considered more scientifically sound if the study had been done over a longer period, the ability to replicate the results of intervention using this simple method has been valuable in helping to improve hand hygiene compliance and thereby reduce hospital-acquired infections.

\section{Conclusion}

This study shows that hand hygiene compliance can be improved by using the WHO five-step multimodal approach and appointing champions for an area. This could reduce hospital-acquired infections and decrease antibiotic resistance, which would greatly improve patient care and reduce the future cost of healthcare. The role of the ward champion can be extended to an infection prevention and control link programme, since such improvements must be sustained through behaviour and a culture change management process, which could also provide a model for further research.

\section{References}

1. Kampf G, Kramer A. Epidemiologic background of hand hygiene and evaluation of the most important agents for scrubs and rubs. Clin Microbiol Rev 2004;17(4):863-893. DOI: 10.1128/CMR.17.4.863893.2004

2. Brink A, Feldman C, Duse A, et al. Guidelines for the management of nosocomial infections in South Africa. S Afr J Epidemiol Infect 2006;21(4):152-160.

3. Whitelaw A. Hospital acquired infections: A review. Health 24, 2014. http://www.health24.com/ Medical/Diseases/Hospital-Acquired-Infections-20120721 (accessed 5 January 2015).

Boyce JM, Pittet D. Guideline for hand hygiene in health-care settings. Recommendations of the healthcare infection control practices advisory committee and the HICPAC/ SHEA/ APIC/IDSA Hand Hygiene task force. MMWR Morb Mortal Wkly Rep 2002;51(RR-16):1- 45.

5. Lam BCC, Lee J, Lau YL. Hand hygiene practices in a neonatal intensive care unit: A multimodal 5. Lam BCC, Lee J, Lau YL. Hand hygiene practices in a neonatal intensive care unit: A multimodal
intervention and impact on nosocomial infection. Pediatrics 2004;114(5):565-571. DOI:10.1542/ peds.2004-1107

peds.2004-1107
6. Pittet D. Improving adherence to hand hygiene practice: A multidisciplinary approach. Emerg Infect 6. Pittet D. Improving adherence to hand hygiene practice
Dis 2001;7(2):234-240. DOI:10.3201/eid0702.700234

7. Babiarz LS, Savoie B, McGuire M, McConnell L, Nagy P. Hand sanitizer-dispensing door handles increase hand hygiene compliance: A pilot study. Am J Infect Control 2014;42(4):443-445. DOI:10.1016/j.ajic.2013.11.009

8. Erasmus V, Daha TJ, Brug H, et al. Systematic review of studies on compliance with hand hygiene guidelines in hospital care. Infect Control Hospital Epidemiol 2010;31(3):283-294. DOI:10.1086/650451

9. Mestre G, Berbel C, Tortajada P, et al. 'The 3/3 strategy': A successful multifaceted hospital wide hand hygiene intervention based on WHO and continuous improvement methodology. PLoS One 2012;7(10):1-12. DOI:10.1371/journal.pone.0047200

10. Al-Tawfiq JA, Abed MS, Al-Yami N, Birrer RB. Promoting and sustaining a hospital-wide, multifaceted hand hygiene program resulted in significant reduction in health care-associated infections. Am J Infect Control 2013;41(6):482-486. DOI:10.1016/j.ajic.2012.08.009

11. World Health Organization. The WHO guidelines on hand hygiene in health care. 2009. http://apps.

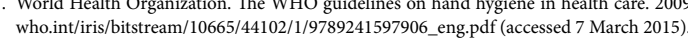

12. Bischoff WE, Reynolds TM, Sessler CN, Edmond MB, Wentzel RP. 2000. Handwashing compliance by health care workers: The impact of introducing an accessible, alcohol-based hand antiseptic. Arch
are Intern Med 2000;160(7):1017-1021. DOI: 10.1001/archinte.160.7.1017

Accepted 14 February 2016. 
Appendix 1. Educational posters placed above the basins in the wards

\section{YOUR FIVE MOMENTS FOR}
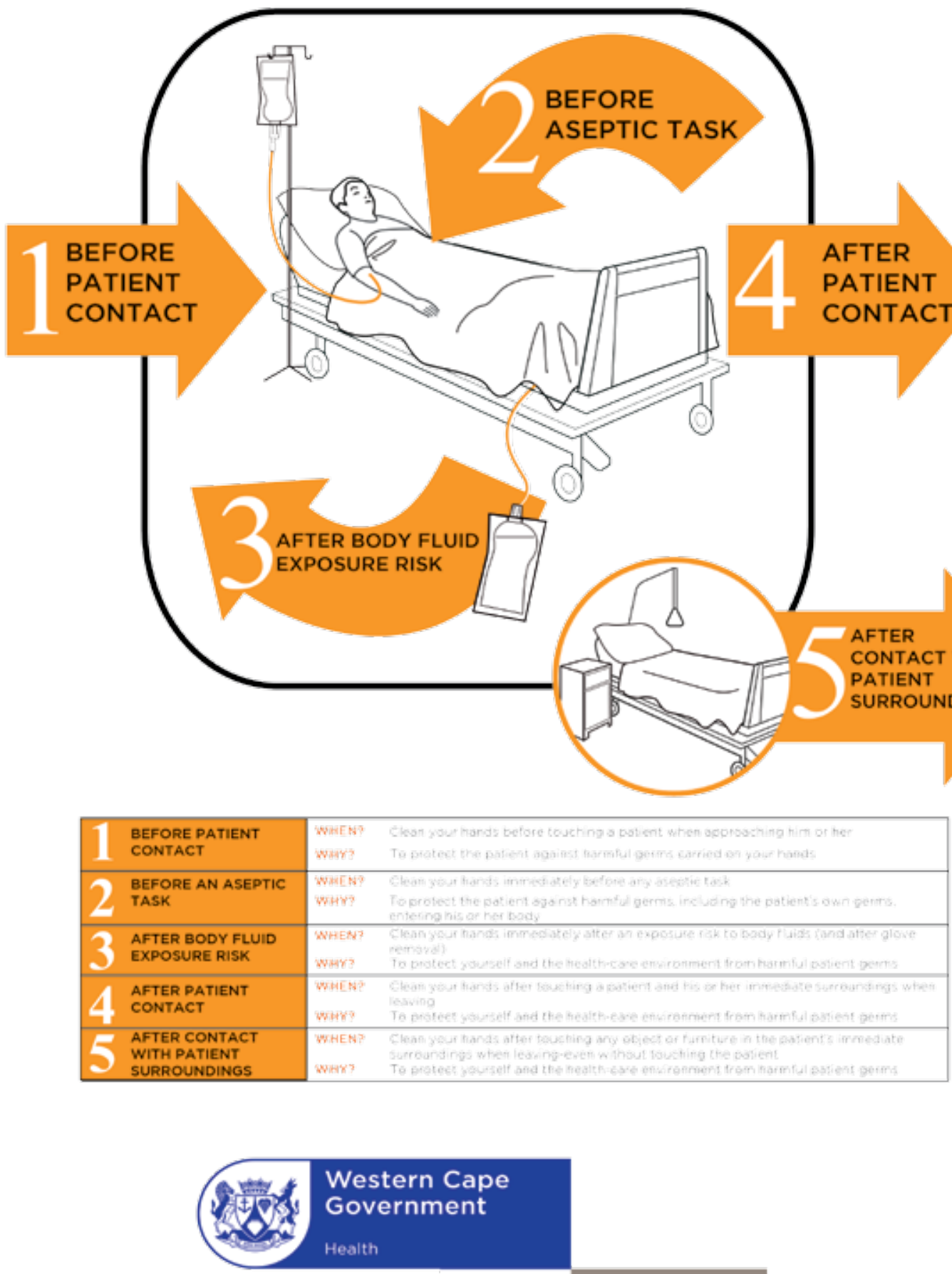

BETTER TOGETHER. 


\section{HOW TO HAND WASH?}

WASH HANDS ONLY WHEN VISIBLY SOILED! OTHERWISE, USE HANDRUB!

(1)

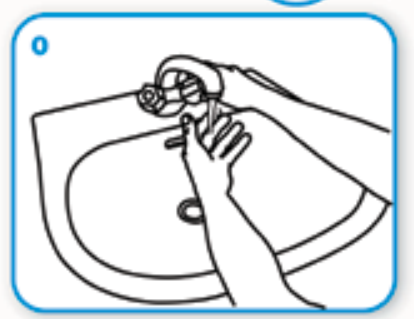

Wet hands with water

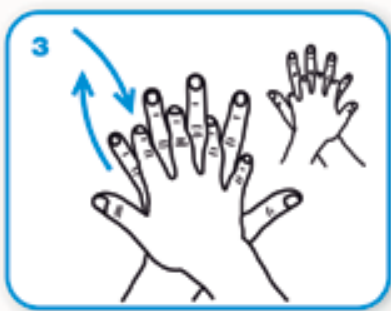

Right palm over left

dorsum with interlaced

fingers and vice versa

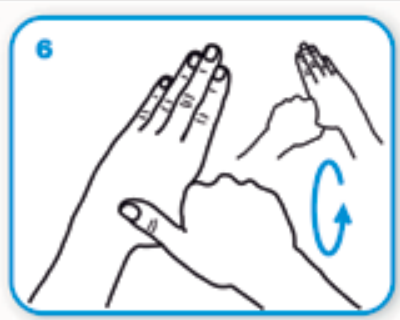

Rotational rubbing of left thumb clasped in right palm and vice versa

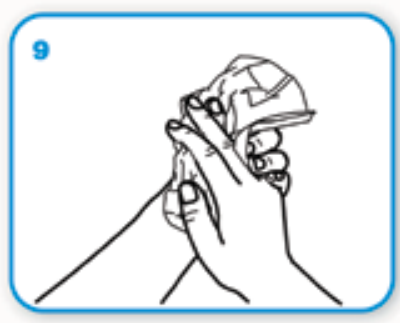

Dry thoroughly with a single use towel

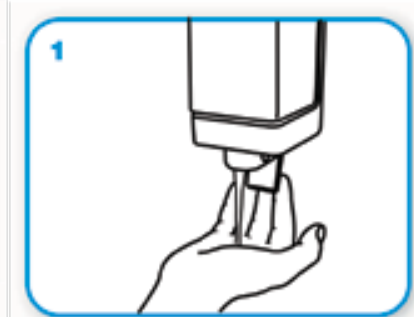

Apply enough soap to cover all hand surfaces

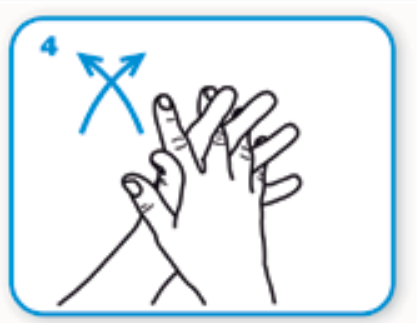

Palm to paim with fingers interlaced

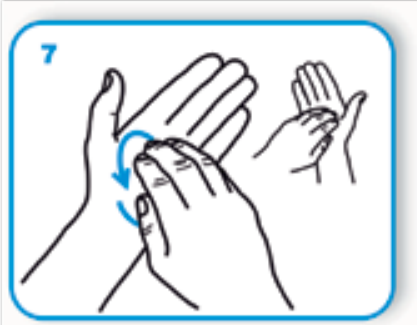

Rotational rubbing.

backwards and forwards

with clasped fingers of

right hand in left palm and vice versa

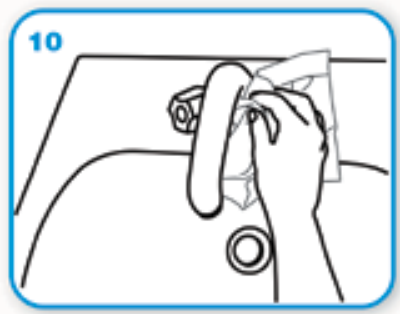

Use towel to turn off faucet

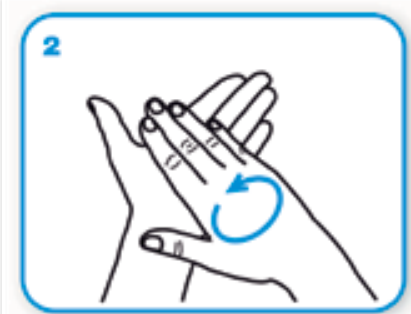

Rub hands palm to palm

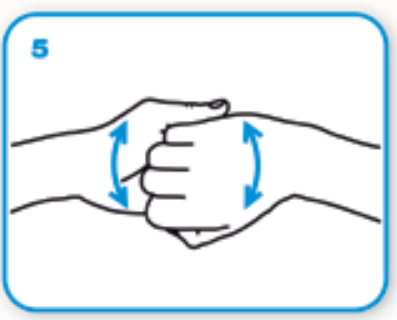

Backs of fingers to

opposing palms with fingers interlocked

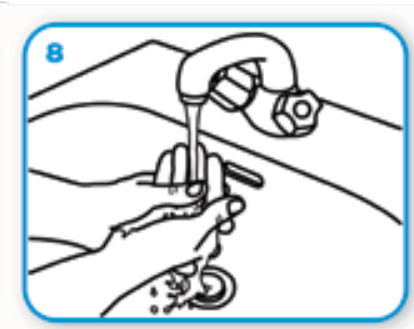

Rinse hands with water

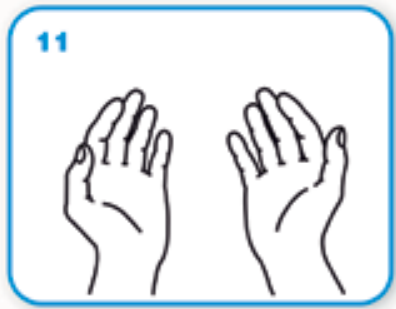

...and your hands are safe. 


\section{HOW TO HANDRUB?}

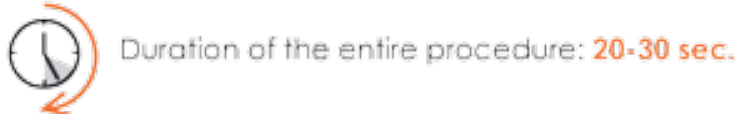
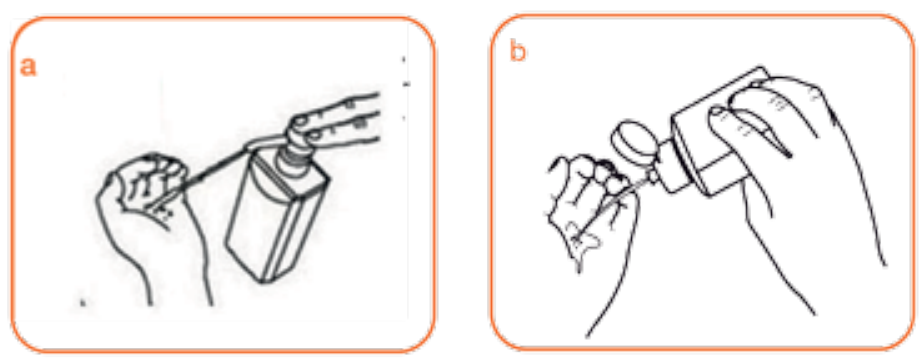

Apply a palmful of the product in a cupped hand and cover all surfaces.

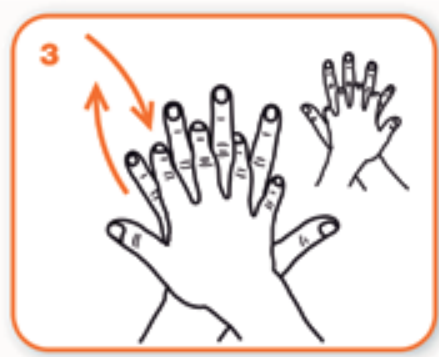

Right palm over left dorsum with interlaced fingers and vice versa

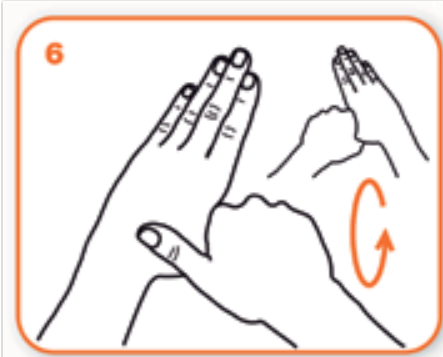

Rotational rubbing of left thumb clasped in right palm and vice versa

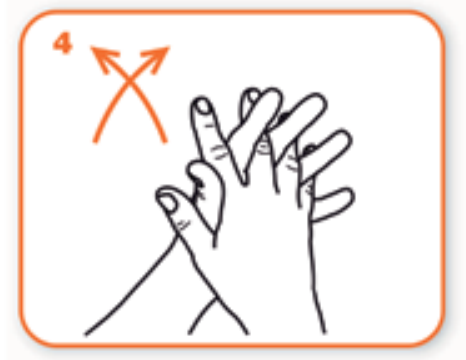

Palm to palm with interlaced fingers

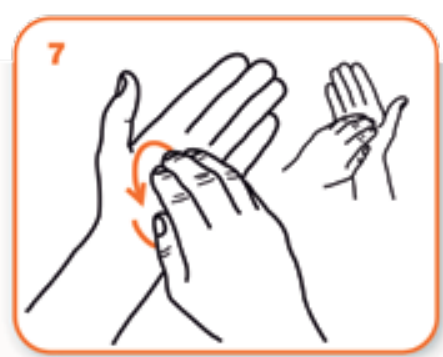

Rotational rubbing, backwards and forwards with clasped fingers of right hand in left palm and vice versa

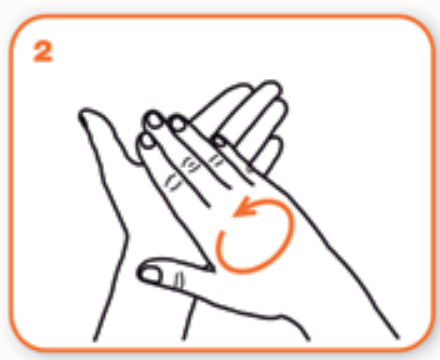

Rub hands palm to polm

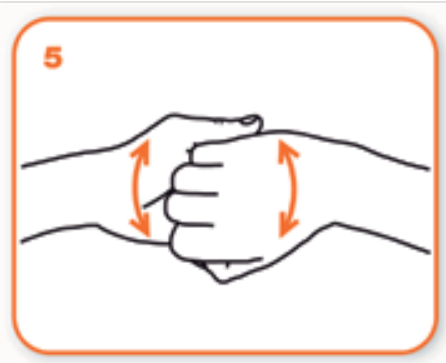

Backs of fingers to opposing palms with fingers interlocked

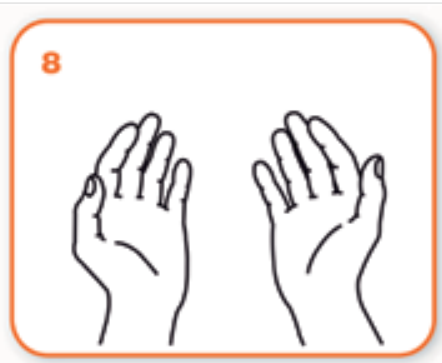

.... once dry, your hands are safe

\section{Western Cape}

Government 